\title{
Microwave-assisted, one-pot multicomponent synthesis of highly substituted pyridines using KF/alumina
}

\author{
Krishna Nand Singh* and Satish Kumar Singh \\ Department of Chemistry, Faculty of Science, Banaras Hindu University, \\ Varanasi-221005, India \\ E-mail: knsinghbhu@yahoo.co.in
}

\begin{abstract}
$\mathrm{KF} /$ alumina in conjunction with mono-mode microwave irradiation brings about an efficient one-pot synthesis of substituted pyridines in high yields using a multicomponent reaction of aromatic aldehydes, malononitrile, and thiophenol in ethanol. The effect of different bases on the product yield has also been investigated under conventional heating as well as under microwave conditions by varying power (Watt), temperature and time.
\end{abstract}

Keywords: Microwave, multicomponent synthesis, pyridines, heterogeneous catalyst, privileged medicinal scaffold

\section{Introduction}

Microwave (MW) provides a powerful way to do synthetic chemistry in the light of the current paradigm shift to "Green Chemistry". It provides many chemical reactions with attributes, such as enhanced reaction rates, higher yields of pure products, better selectivity, improved ease of manipulation, rapid optimization of reactions and several ecofriendly advantages. ${ }^{1}$ Multicomponent reactions (MCRs) have recently taken a new dimention in organic synthesis, as they comply well with the requirements for ideal organic syntheses. ${ }^{2}$ According to the current synthetic requirements, environmentally benign multicomponent procedures employing microwave methodology are particularly welcome.

The usefulness of MCRs is even greater when they provide access to "privileged medicinal scaffolds". ${ }^{3}$ One such significant scaffold is the pyridine nucleus which is key constituent of a wide range of both natural and synthetic bioactive compounds. ${ }^{4}$ Owing to its vast medicinal utility, various methods have been adopted for the preparation of substituted pyridines, viz., hetero-Diels-Alder reaction of 3-siloxy-1-aza-1,3-butadiens and 2H-1,4-oxazinones with acetylenes, ${ }^{5}$ ruthenium-catalyzed cycloisomerization of 3-azadienynes, ${ }^{6}$ Mannich reaction of aldehydes and iminium salts, ${ }^{7}$ Vilsmeier-Haack reaction of $\alpha$-hydroxyketenedithioacetals, ${ }^{8} 6 \pi$ - 
azaelectrocyclization of azatrienes, catalytic oxidation of 1,4-dihydropyridines by $\mathrm{RuCl}_{3} / \mathrm{O}_{2}{ }^{9}$, [4+2] cycloadditions of oximinosulfonates, ${ }^{10}$ conversion of conjugated oximes under Vilsmeier conditions, ${ }^{11} \mathrm{~N}$-methylene-tert-butylamine with enamines, ${ }^{12}$ conversion of ketene dithioacetals to substituted pyridines ${ }^{13}$ and multicomponent reactions using triethyl amine/DABCO ${ }^{14}$ $[\mathrm{bmIm}] \mathrm{OH}^{15}, \mathrm{DBU}^{16}$ and $\mathrm{ZnCl}_{2}{ }^{17}$. Many of these methods, however, require high temperature and suffer from the serious limitations like formation of considerable amounts of side products and lower product yields. Subsequently, there stands a demand and scope for an efficient, facile and eco-safe approach.

Organic reactions promoted by a solid heterogeneous catalyst have attracted widespread interest and are advantageous because of operational simplicity, high selectivity, and clean separation of the product. Potassium fluoride impregnated over alumina (KF/alumina) has been recognized as a remarkably useful green heterogeneous catalyst to promote a wide range of organic reactions. ${ }^{18}$ We herein report a rapid and green approach to achieve highly substituted pyridines in excellent yields in the presence of catalytic amount of KF/alumina under controlled MW irradiation.

\section{Results and Discussion}

In view of the potential medicinal importance of the products and considering the limitations of the existing methods, we have investigated a KF/alumina (10 mol\%) catalyzed, one-pot, simple and efficient procedure for the rapid construction of substituted pyridines via a three-component reaction of aldehydes, malononitrile and thiophenols (molar ratio 1:2:1) in ethanol under reflux (56-82 \%) and also under controlled microwave conditions (62-93\%) (Scheme 1).

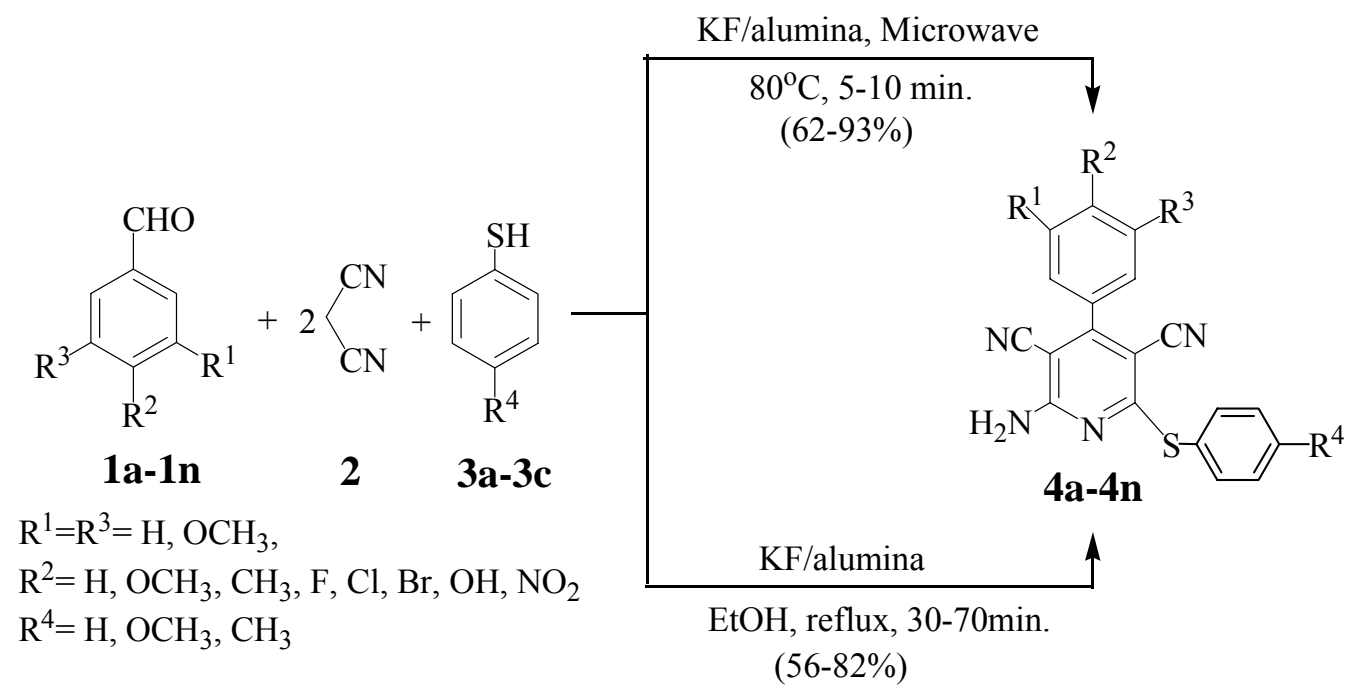

\section{Scheme 1}


The observed synthesis of substituted pyridines works well for unsubstituted as well as for electron-rich/electron-deficient aromatic aldehydes. In case of aliphatic and heterocyclic aldehydes, however, some intractable products of unidentified nature were obtained. In order to optimize the reaction conditions, a typical reaction of benzaldehyde (1a, $1 \mathrm{mmol})$, malononitrile ( $2 \mathrm{mmol})$ and thiophenol $(1 \mathrm{mmol})$ was carried out in the presence of different bases under conventional heating as well as under microwave irradiation at different power (Watt), temperature and time in ethanol. The outcome is presented in Table 1. It is evident from the table that a catalytic amount of KF/alumina accomplishes the reaction successfully and the use of the microwave irradiation further enhances the yield of the product considerably with dramatic reduction in the reaction time, the best result being obtained using $120 \mathrm{~W}$ at $80^{\circ} \mathrm{C}$ in 5 minutes in the presence of KF/alumina (10 mol\%) (cf. entry 6).

Table 1. Optimization of reaction conditions using compound 1a as reference

\begin{tabular}{ccccccccc}
\hline Entry & $\begin{array}{c}\text { Base } \\
(10 \text { mol\%) }\end{array}$ & \multicolumn{4}{c}{ Microwave } & \multicolumn{4}{c}{ Conventional } \\
\cline { 3 - 8 } & & $\begin{array}{c}\text { MW } \\
(\text { Watt })\end{array}$ & $\begin{array}{c}\text { Temp. } \\
\left({ }^{\circ} \mathrm{C}\right)\end{array}$ & $\begin{array}{c}\text { Time } \\
(\text { min. })\end{array}$ & $\begin{array}{c}\text { Yield } \\
(\%)\end{array}$ & $\begin{array}{c}\text { Temp. } \\
\left({ }^{\circ} \mathrm{C}\right)\end{array}$ & $\begin{array}{c}\text { Time } \\
(\mathrm{h})\end{array}$ & $\begin{array}{c}\text { Yield } \\
(\%)\end{array}$ \\
\hline 1 & Basic alumina & 120 & 80 & 15 & Nil & RT & 3 & Nil \\
2 & Basic alumina & 180 & 100 & 15 & Nil & 78 & 3 & Nil \\
3 & $\mathrm{KF}$ & 100 & 80 & 7 & 46 & RT & 2 & 20 \\
4 & $\mathrm{KF}$ & 120 & 80 & 5 & 51 & 78 & 1.5 & 43 \\
5 & $\mathrm{KF} /$ alumina & 100 & 80 & 5 & 74 & $\mathrm{RT}$ & 2 & 36 \\
$\mathbf{6}$ & $\mathrm{KF} /$ alumina & $\mathbf{1 2 0}$ & $\mathbf{8 0}$ & $\mathbf{5}$ & $\mathbf{8 6}$ & $\mathbf{7 8}$ & $\mathbf{0 . 7 5}$ & $\mathbf{7 1}$ \\
7 & $\mathrm{KF} /$ alumina & 120 & 100 & 5 & 85 & - & - & - \\
8 & $\mathrm{KF} /$ alumina & 180 & 100 & 5 & 84 & - & - & - \\
9 & $\mathrm{~K} \mathrm{CO}_{3}$ & 180 & 100 & 15 & Nil & 78 & 3 & Nil \\
10 & $\mathrm{NaOH}$ & 180 & 100 & 15 & Nil & 78 & 3 & Nil \\
\hline
\end{tabular}

Under the optimized set of reaction conditions (entry 6), a number of aromatic aldehydes 1 were allowed to undergo multicomponent reaction with malononitrile $\mathbf{2}$ and thiophenol $\mathbf{3}$ in a molar ratio of 1:2:1 with $\mathrm{KF} /$ alumina $(10 \mathrm{~mol} \%)$ in ethanol under reflux $\left(78{ }^{\circ} \mathrm{C}\right)$ as well as under microwave $\left(120 \mathrm{~W}, 80^{\circ} \mathrm{C}\right)$ heating. After completion of the reaction, the resulting precipitate was filtered and recrystallized from acetonitrile/methanol to yield pure substituted pyridine $4 \mathbf{4}-\mathbf{4 n}$. All the products were crystalline and fully characterized based on their melting points, elemental analyses and spectral data (IR, ${ }^{1} \mathrm{HNMR},{ }^{13} \mathrm{CNMR}$ ). The reaction data are indicated in Table 2 and reveal that the aldehydes containing electron withdrawing groups undergo reaction sluggishly with diminution of the product yield. 
Table 2. KF/alumina catalyzed three-component reaction of substituted aldehydes with malononitrile and thiols

\begin{tabular}{|c|c|c|c|c|c|c|c|c|}
\hline \multirow{3}{*}{ Entry } & \multirow{3}{*}{$\mathrm{R}^{1}$} & \multirow{3}{*}{$\mathrm{R}^{2}$} & \multirow{3}{*}{$\mathrm{R}^{3}$} & \multirow{3}{*}{$\mathrm{R}^{4}$} & \multicolumn{4}{|c|}{ Reaction Conditions } \\
\hline & & & & & \multicolumn{2}{|c|}{ Microwave $^{b}$} & \multicolumn{2}{|c|}{ Conventional } \\
\hline & & & & & $\begin{array}{l}\text { Time } \\
\text { (min.) }\end{array}$ & $\begin{array}{l}\text { Yield } \\
(\%)^{\mathrm{a}}\end{array}$ & $\begin{array}{l}\text { Time } \\
(\min .)\end{array}$ & $\begin{array}{l}\text { Yield } \\
(\%)^{\mathrm{a}}\end{array}$ \\
\hline 1. & $\mathrm{H}$ & $\mathrm{H}$ & $\mathrm{H}$ & $\mathrm{H}$ & 5 & 86 & 45 & 71 \\
\hline 2. & $\mathrm{H}$ & $\mathrm{H}$ & $\mathrm{H}$ & $\mathrm{CH}_{3}$ & 5 & 90 & 40 & 78 \\
\hline 3. & $\mathrm{H}$ & $\mathrm{H}$ & $\mathrm{H}$ & $\mathrm{OCH}_{3}$ & 5 & 92 & 35 & 82 \\
\hline 4. & $\mathrm{H}$ & $\mathrm{OCH}_{3}$ & $\mathrm{H}$ & $\mathrm{H}$ & 5 & 92 & 30 & 81 \\
\hline 5. & $\mathrm{H}$ & $\mathrm{OCH}_{3}$ & $\mathrm{H}$ & $\mathrm{OCH}_{3}$ & 5 & 93 & 30 & 82 \\
\hline 6. & $\mathrm{H}$ & $\mathrm{NO}_{2}$ & $\mathrm{H}$ & $\mathrm{H}$ & 10 & 62 & 70 & 56 \\
\hline 7. & $\mathrm{H}$ & $\mathrm{Cl}$ & $\mathrm{H}$ & $\mathrm{H}$ & 7 & 83 & 60 & 68 \\
\hline 8. & $\mathrm{Cl}$ & $\mathrm{H}$ & $\mathrm{H}$ & $\mathrm{H}$ & 7 & 81 & 60 & 67 \\
\hline 9. & $\mathrm{H}$ & $\mathrm{F}$ & $\mathrm{H}$ & $\mathrm{H}$ & 7 & 79 & 60 & 62 \\
\hline 10 & $\mathrm{H}$ & $\mathrm{Br}$ & $\mathrm{H}$ & $\mathrm{H}$ & 7 & 87 & 50 & 71 \\
\hline 11. & $\mathrm{Br}$ & $\mathrm{H}$ & $\mathrm{H}$ & $\mathrm{H}$ & 7 & 85 & 60 & 69 \\
\hline 12. & $\mathrm{H}$ & $\mathrm{CH}_{3}$ & $\mathrm{H}$ & $\mathrm{H}$ & 5 & 90 & 30 & 79 \\
\hline 13. & $\mathrm{OCH}_{3}$ & $\mathrm{OH}$ & $\mathrm{H}$ & $\mathrm{H}$ & 5 & 80 & 60 & 65 \\
\hline 14. & $\mathrm{OCH}_{3}$ & $\mathrm{OCH}_{3}$ & $\mathrm{OCH}_{3}$ & $\mathrm{H}$ & 7 & 88 & 50 & 70 \\
\hline
\end{tabular}

${ }^{\mathrm{a}}$ Isolated yield based on aldehydes.

${ }^{\mathrm{b}}$ Microwave heating performed on 120 Watt power and $80{ }^{\circ} \mathrm{C}$ temperature.

\section{Conclusions}

The present work describes an efficient one-pot multicomponent synthesis of 2-amino-3,5dicarbonitrile-6-sulfanylpyridines through a condensation of aldehydes, malononitrile and thiols in ethanol under controlled microwave as well as conventional heating conditions using $\mathrm{KF} / \mathrm{alumina}$ as a heterogeneous green catalyst.

\section{Experimental Section}

General. All the chemicals were procured from Aldrich, USA, and E. Merck, Germany and were purified prior to their use. IR spectra were recorded on a JASCO FT/IR-5300 spectrophotometer. NMR spectra were run on a JEOL AL300 FTNMR spectrometer; chemical shifts are given in $\delta$ ppm, relative to TMS as internal standard. Elemental microanalysis was performed on Exeter Analytical Inc Model CE-440 CHN Analyzer. Melting points were measured in open capillaries 
and are uncorrected. The microwave irradiation was effected using the CEM's Discover Bench Mate single-mode microwave synthesis system using safe pressure regulation $10-\mathrm{mL}$ pressurized vials with "snap-on" cap. KF/Alumina was prepared according to a method described in literature. $^{18}$

\section{General conventional procedure for synthesis of polysubstituted pyridines $\mathbf{4}$}

To a mixture of aldehyde $(1.5 \mathrm{mmol})$ and malononitrile $(3 \mathrm{mmol})$ in anhydrous ethanol $(3 \mathrm{~mL})$ was added $\mathrm{KF} /$ alumina $(10 \mathrm{~mol} \%)$ and the resulting mixture was stirred at room temperature. A precipitation took place within 2-5 minutes, after which thiophenol (1.5 mmol) was added while continuing the stirring. The reaction mixture was subsequently reflux for 30-70 minutes to complete the reaction (TLC) and then allowed to cool at room temperature. The resulting precipitate was filtered and recrystallized from acetonitrile/methanol to yield pure product $\mathbf{4 a - 4 n}$.

\section{General microwave procedure for synthesis of polysubstituted pyridines 4}

Aldehyde (1 mmol), malononitrile (2 mmol), KF/alumina (10 mol\%), thiophenol $(1 \mathrm{mmol})$ and anhydrous ethanol $(1.5 \mathrm{~mL})$ were mixed and placed in a sealed pressure regulation $10-\mathrm{mL}$ pressurized vials with "snap-on" cap and was irradiated in the single-mode microwave synthesis system at $120 \mathrm{~W}$ power and $80^{\circ} \mathrm{C}$ temperature for 5-10 minutes. After the completion of reaction (TLC), the mixture was cooled and precipitate formed was filtered and recrystallized from acetonitrile/methanol to yield the pure product $\mathbf{4 a - 4 n}$.

2-Amino-4-phenyl-6-phenylsulfanyl-pyridine-3,5-dicarbonitrile 4a. Colourless solid, mp: 215-217 ${ }^{\circ} \mathrm{C}$ (Lit. ${ }^{15} \mathrm{mp}: 216-218^{\circ} \mathrm{C}$ ). IR (KBr): 3475, 3360, 2925, 2212, 1619, 1544, 1461, 1254 , 1154, 1078, $750 \mathrm{~cm}^{-1}$. ${ }^{1} \mathrm{H}$ NMR (300 MHz, DMSO-d $\left.\mathrm{d}_{6}\right): \delta=7.8\left(\mathrm{bs}, 2 \mathrm{H}, \mathrm{NH}_{2}\right), 7.51-7.59(\mathrm{~m}$, $10 \mathrm{H}, \mathrm{Ar}-\mathrm{H}) .{ }^{13} \mathrm{C}$ NMR $\left(75 \mathrm{MHz}, \mathrm{DMSO}-\mathrm{d}_{6}\right): \delta=167.6,161.0,158.7,136.1,134.2,130.9,130.1$, 129.7, 129.2, 128.6, 127.8, 116.1, 94.5, 87.4. Anal. Calcd for $\mathrm{C}_{19} \mathrm{H}_{12} \mathrm{~N}_{4} \mathrm{~S}$ (328.39): C, 69.49; H, 3.69; N, 17.06. Found: C, 69.24; H, 3.58; N, 17.17.

2-Amino-6-(4-methylphenylsulfanyl)-4-phenyl-pyridine-3,5-dicarbonitrile 4b. Light-Yellow solid, mp: 218-220 ${ }^{\circ} \mathrm{C}$. IR (KBr): 3452, 3323, 3209, 3056, 2922, 2215, 1617, 1545, 1452, 1310, 1181, 1020, 754, $704 \mathrm{~cm}^{-1} .{ }^{1} \mathrm{H}$ NMR (300 MHz, DMSO-d 6 ): $\delta=7.78$ (bs, 2H, NH ), 7.46-7.56 $(\mathrm{m}, 6 \mathrm{H}, \mathrm{Ar}-\mathrm{H}), 7.29-7.32(\mathrm{~m}, 3 \mathrm{H}, \mathrm{Ar}-\mathrm{H}), 2.3\left(\mathrm{~s}, 3 \mathrm{H}, \mathrm{CH}_{3}\right) .{ }^{13} \mathrm{C}$ NMR $\left(75 \mathrm{MHz}, \mathrm{DMSO}-\mathrm{d}_{6}\right): \delta=$ $166.6,159.6,158.6,139.6,135.0,133.9,130.3,130.1,128.7,128.4,123.4,115.3,115.0$, 93.1, 86.9, 20.9. Anal. Calcd for $\mathrm{C}_{20} \mathrm{H}_{14} \mathrm{~N}_{4} \mathrm{~S}$ (342.42): C, 70.15; H, 4.12; N, 16.36. Found: C, 70.28; H, $4.19 ; \mathrm{N}, 16.47$.

2-Amino-6-(4-methoxyphenylsulfanyl)-4-phenyl-pyridine-3,5-dicarbonitrile (4c). Colourless solid, mp: 228-229 ${ }^{\circ} \mathrm{C}$. IR (KBr): 3432, 3332, 3222, 3057, 2676, 2210, 1629, 1540, 1417, 1256 , $1177,1028,799,754,708 \mathrm{~cm}^{-1}$. ${ }^{1} \mathrm{H}$ NMR (300 MHz, DMSO-d 6 ): $\delta=7.75\left(\mathrm{bs}, 2 \mathrm{H}, \mathrm{NH}_{2}\right), 7.49-$ $7.56(\mathrm{~m}, 6 \mathrm{H}, \mathrm{Ar}-\mathrm{H}), 7.04-7.07$ (m, 3H, Ar-H), $3.82\left(\mathrm{~s}, 3 \mathrm{H}, \mathrm{OCH}_{3}\right) .{ }^{13} \mathrm{C}$ NMR $(75 \mathrm{MHz}, \mathrm{DMSO}-$ $\left.\mathrm{d}_{6}\right): \delta=166.4,161.2,160.0,158.7,135.3,130.7,130.1,129.9,127.6,126.3,115.9,114.7,93.7$, 
87.5, 55.4. Anal. Calcd for $\mathrm{C}_{20} \mathrm{H}_{14} \mathrm{~N}_{4} \mathrm{OS}$ (358.42): C, 67.02; H, 3.94; N, 15.63. Found: C, 67.17; $\mathrm{H}, 3.88 ; \mathrm{N}, 15.71$.

2-Amino-4-(4-methoxyphenyl)-6-phenylsulfanyl-pyridine-3,5-dicarbonitrile 4d. Colourless solid, mp: $239-241{ }^{\circ} \mathrm{C}$ (Lit. ${ }^{15} \mathrm{mp}: 238-240{ }^{\circ} \mathrm{C}$ ). IR (KBr): 3439, 3330, 3226, 2852, 2218, 1641 , 1545, 1513, 1257, 1185, 1021, $755 \mathrm{~cm}^{-1} .{ }^{1} \mathrm{H}$ NMR (300 MHz, DMSO-d $): \delta=7.72(\mathrm{bs}, 2 \mathrm{H}$, $\left.\mathrm{NH}_{2}\right), 7.48-7.59(\mathrm{~m}, 6 \mathrm{H}, \mathrm{Ar}-\mathrm{H}), 7.09-7.12(\mathrm{~m}, 3 \mathrm{H}, \mathrm{Ar}-\mathrm{H}), 3.83\left(\mathrm{~s}, 3 \mathrm{H}, \mathrm{OCH}_{3}\right) .{ }^{13} \mathrm{C} \mathrm{NMR}(75$ MHz, DMSO-d $\left.{ }_{6}\right): \delta=166.1,160.8,159.7,158.2,134.7,130.2,129.6,129.4,127.2,125.7$, 115.5, 115.2, 114.0, 93.3, 86.9, 55.3. Anal. Calcd for $\mathrm{C}_{20} \mathrm{H}_{14} \mathrm{~N}_{4} \mathrm{OS}$ (358.42): C, 67.02; H, 3.94; N, 15.63. Found: C, 66.83; H, 3.91; N, 15.75.

2-Amino-4-(4-methoxyphenyl)-6-(4-methoxyphenylsulfanyl)-pyridine-3,5-dicarbonitrile 4e. White solid, mp: $218-220^{\circ} \mathrm{C}$. IR (KBr): 3399, 3321, 3222, 2937, 2840, 2213, 1642, 1545, 1507 , 1460, 1251, 1174, 1027, 825, $677 \mathrm{~cm}^{-1} .{ }^{1} \mathrm{H}$ NMR (300 MHz, DMSO-d 6 ): $\delta=7.69\left(\mathrm{bs}, 2 \mathrm{H}, \mathrm{NH}_{2}\right)$, 7.48-7.52 (m, 4H, Ar-H), 7.04-7.13 (m, 4H, Ar-H), $3.85\left(\mathrm{~s}, 3 \mathrm{H}, \mathrm{OCH}_{3}\right), 3.82\left(\mathrm{~s}, 3 \mathrm{H}, \mathrm{OCH}_{3}\right) .{ }^{13} \mathrm{C}$ NMR (75 MHz, DMSO-d 6 ): $\delta=167.3,160.9,160.6,159.8,158.2,136.1,125.8,117.2,115.6$, 115.4, 115.1, 114.1, 92.9, 86.7, 55.4, 55.3. Anal. Calcd for $\mathrm{C}_{21} \mathrm{H}_{16} \mathrm{~N}_{4} \mathrm{O}_{2} \mathrm{~S}$ (388.44): C, 64.93; $\mathrm{H}$, 4.15; N, 14.42. Found: C, 64.77; H, 4.21; N, 14.54 .

2-Amino-4-(4-nitrophenyl)-6-phenylsulfanyl-pyridine-3,5-dicarbonitrile 4f. Yellow solid, mp: 286-288 ${ }^{\circ} \mathrm{C}$ (Lit. ${ }^{15} \mathrm{mp}: 287-289^{\circ} \mathrm{C}$ ). IR (KBr): 3420, 3325, 3234, 3083, 2223, 2210, 1637 , 1543, 1530, 1456, 1422, 1352, 1256, 1167, 890, 846, 756, $\mathrm{cm}^{-1}$. ${ }^{1} \mathrm{H}$ NMR (300 MHz, DMSO-d 6 ): $\delta=8.38(\mathrm{~d}, 2 \mathrm{H}, \mathrm{Ar}-\mathrm{H}), 8.10\left(\mathrm{bs}, 2 \mathrm{H}, \mathrm{NH}_{2}\right), 7.86(\mathrm{~d}, 2 \mathrm{H}, \mathrm{Ar}-\mathrm{H}), 7.58-7.60(\mathrm{~m}, 2 \mathrm{H}, \mathrm{Ar}-\mathrm{H}), 7.43-$ $7.46(\mathrm{~m}, 3 \mathrm{H}, \mathrm{Ar}-\mathrm{H}) .{ }^{13} \mathrm{C} \mathrm{NMR}\left(75 \mathrm{MHz}, \mathrm{DMSO}-\mathrm{d}_{6}\right): \delta=166.2,159.7,156.7,148.8,140.2$, $134.9,130.3,130.1,129.7,124.0,115.2,114.6$, 93.0, 86.9. Anal. Calcd for $\mathrm{C}_{19} \mathrm{H}_{11} \mathrm{~N}_{5} \mathrm{O}_{2} \mathrm{~S}$ (373.39): C, 61.12; H, 2.97; N, 18.76. Found: C, 60.85; H, 3.07; N, 18.91.

2-Amino-4-(4-chlorophenyl)-6-phenylsulfanyl-pyridine-3,5-dicarbonitrile 4g. Colourless solid, mp: $223-22{ }^{\circ} \mathrm{C}$ (Lit. ${ }^{15} \mathrm{mp}: 222-224{ }^{\circ} \mathrm{C}$ ). IR (KBr): 3472, 3346, 3221, 2953, 2216, 1630, 1552, 1486, 1257, 1112, $785 \mathrm{~cm}^{-1} .{ }^{1} \mathrm{H}$ NMR (300 MHz, DMSO-d 6 ): $\delta=7.76\left(\mathrm{bs}, 2 \mathrm{H}, \mathrm{NH}_{2}\right)$, 7.61-7.69 (m, 6H, Ar-H), 7.41-7.44 (m, 3H, Ar-H). ${ }^{13} \mathrm{C}$ NMR (75 MHz, DMSO-d 6 ): $\delta=166.3$, $159.8,157.5,135.4,135.1,132.9,130.7,129.5,129.3,129.0,126.9,115.3,115.0,93.4,87.3$. Anal. Calcd for $\mathrm{C}_{19} \mathrm{H}_{11} \mathrm{ClN}_{4} \mathrm{~S}$ (362.84): C, 62.89; H, 3.06; N, 15.44. Found: C, 63.03; H, 2.97; N, 15.35 .

2-Amino-4-(3-chlorophenyl)-6-phenylsulfanyl-pyridine-3,5-dicarbonitrile 4h. Colourless solid, mp: $247-249{ }^{\circ} \mathrm{C}$. IR (KBr): 3452, 3344, 3222, 3068, 2218, 1628, 1551, 1528, 1476, 1257 , 1021, 785, $708 \mathrm{~cm}^{-1} .{ }^{1} \mathrm{H}$ NMR (300 MHz, DMSO-d $): \delta=7.71$ (bs, 2H, NH$), 7.67-7.69(\mathrm{~m}, 2 \mathrm{H}$, Ar-H), 7.63 (s, 1H, Ar-H), 7.43-7.47 (m, 6H, Ar-H). Anal. Calcd for $\mathrm{C}_{19} \mathrm{H}_{11} \mathrm{ClN}_{4} \mathrm{~S}$ (362.84): C, 62.89; H, 3.06; N, 15.44. Found: C, 63.07; H, 3.10; N, 15.55.

2-Amino-4-(4-fluorophenyl)-6-phenylsulfanyl-pyridine-3,5-dicarbonitrile 4i. Colourless solid, mp: $218-220^{\circ} \mathrm{C}$ (Lit. ${ }^{17} \mathrm{mp}: 221-22{ }^{\circ} \mathrm{C}$ ). IR (KBr): 3489, 3340, 3224, 2923, 2217, 1631, $1551,1509,1425,1259,1156,1022,825,758 \mathrm{~cm}^{-1} .{ }^{1} \mathrm{H}$ NMR $\left(300 \mathrm{MHz}, \mathrm{DMSO}-\mathrm{d}_{6}\right): \delta=7.84$ (bs, 2H, $\mathrm{NH}_{2}$ ), 7.71-7.75 (m, 6H, Ar-H), 7.49-7.51 (m, 3H, Ar-H). Anal. Calcd for $\mathrm{C}_{19} \mathrm{H}_{11} \mathrm{FN}_{4} \mathrm{~S}$ (346.38): C, 65.88; H, 3.20; N, 16.17. Found: C, 65.75; H, 3.28; N, 16.28. 
2-Amino-4-(4-bromophenyl)-6-phenylsulfanyl-pyridine-3,5-dicarbonitrile 4j. Light-yellow solid, mp: $232-234^{\circ} \mathrm{C}$ (Lit. ${ }^{16} \mathrm{mp}: 255-257^{\circ} \mathrm{C}$ ). IR (KBr): 3477, 3348, 3218, 3068, 2213, 1631, 1541, 1484, 1419, 1315, 1259, 1076, $799 \mathrm{~cm}^{-1} .{ }^{1} \mathrm{H}$ NMR (300 MHz, DMSO-d 6 ): $\delta=7.63(\mathrm{bs}$, $\left.2 \mathrm{H}, \mathrm{NH}_{2}\right), 7.46-7.54(\mathrm{~m}, 6 \mathrm{H}, \mathrm{Ar}-\mathrm{H}), 7.32-7.39$ (m, 3H, Ar-H). Anal. Calcd for $\mathrm{C}_{19} \mathrm{H}_{11} \mathrm{BrN}_{4} \mathrm{~S}$ (407.29): C, 56.03; H, 2.72; N, 13.76. Found: C, 56.19; H, 2.65; N, 13.65.

2-Amino-4-(3-bromophenyl)-6-phenylsulfanyl-pyridine-3,5-dicarbonitrile $\quad 4 \mathbf{k}$. Colourless solid, mp: $255-257^{\circ} \mathrm{C}$ (Lit. ${ }^{15} \mathrm{mp}: 256-258^{\circ} \mathrm{C}$ ). IR (KBr): 3441, 3337, 3220, 2218, 1627, 1552, 1528, 1475, 1257, 1021, 782, $756 \mathrm{~cm}^{-1} .{ }^{1} \mathrm{H}$ NMR (300 MHz, DMSO-d $)$ : $\delta=7.67\left(\mathrm{bs}, 2 \mathrm{H}, \mathrm{NH}_{2}\right)$, 7.62-7.64 (m, 2H, Ar-H), $7.59(\mathrm{~s}, 1 \mathrm{H}, \mathrm{Ar}-\mathrm{H}), 7.45-7.48(\mathrm{~m}, 6 \mathrm{H}, \mathrm{Ar}-\mathrm{H})$. Anal. Calcd for $\mathrm{C}_{19} \mathrm{H}_{11} \mathrm{BrN}_{4} \mathrm{~S}$ (407.29): C, 56.03; H, 2.72; N, 13.76. Found: C, 56.15; H, 2.81; N, 13.88 .

2-Amino-4-(4-methylphenyl)-6-phenylsulfanyl-pyridine-3,5-dicarbonitrile 4l. Colourless solid, mp: $209-211^{\circ} \mathrm{C}$ (Lit. ${ }^{15} \mathrm{mp}: 208-211^{\circ} \mathrm{C}$ ). IR (KBr): 3472, 3348, 3212, 2913, 2215, 1616, $1538,1512,1301,1257,1118,1020,876,758 \mathrm{~cm}^{-1} .{ }^{1} \mathrm{H}$ NMR $\left(300 \mathrm{MHz}, \mathrm{CDCl}_{3}\right): \delta=7.54-7.57$ $(\mathrm{m}, 3 \mathrm{H}, \mathrm{Ar}-\mathrm{H}), 7.33-7.47$ (m, 6H, Ar-H), 5.44 (bs, 2H, NH$), 2.43\left(\mathrm{~s}, 3 \mathrm{H}, \mathrm{CH}_{3}\right) .{ }^{13} \mathrm{C}$ NMR $(75$ $\left.\mathrm{MHz}, \mathrm{CDCl}_{3}\right): \delta=168.9,159.3,158.5,141.4,135.7,130.2,129.8,129.7,129.3,128.4,127.2$, 115.4, 114.9, 95.8, 87.3, 21.5. Anal. Calcd for $\mathrm{C}_{20} \mathrm{H}_{14} \mathrm{~N}_{4} \mathrm{~S}$ (342.42): C, 70.15; H, 4.12; N, 16.36 . Found: C, 70.03; H, 4.19; N, 16.22.

2-Amino-4-(4-hydroxy-3-methoxyphenyl)-6-phenylsulfanyl-pyridine-3,5-dicarbonitrile $\mathbf{4 m}$. Colourless solid, mp: $217-219{ }^{\circ} \mathrm{C}$ (Lit. ${ }^{15} \mathrm{mp}: 218-220^{\circ} \mathrm{C}$ ). IR (KBr): 3456, 3354, 3222, 2928, 2218, 2197, 1646, 1552, 1431, 1262, 1134, 1030, $770 \mathrm{~cm}^{-1} .{ }^{1} \mathrm{H}$ NMR (300 MHz, DMSO-d 6$): \delta=$ 9.68 (s, 1H, OH), 7.71 (bs, 2H, NH 2$), 7.59$ (d, 2H, Ar-H), 7.16 (s 1H, Ar-H), 6.91-6.98 (m, 5H, Ar-H), 3.81 (s, 3H, $\mathrm{OCH}_{3}$ ). Anal. Calcd for $\mathrm{C}_{20} \mathrm{H}_{14} \mathrm{~N}_{4} \mathrm{O}_{2} \mathrm{~S}$ (374.42): C, 64.16; H, 3.77; N, 14.96 . Found: C, 64.26; H, 3.71; N, 15.07.

2-Amino-6-(phenylsulfanyl)-4-(3,4,5-trimethoxyphenyl)-pyridine-3,5-dicarbonitrile 4n. White solid, mp: $238-239{ }^{\circ} \mathrm{C}$ (Lit. ${ }^{14 \mathrm{a}} \mathrm{mp}: 238-239^{\circ} \mathrm{C}$ ). IR (KBr): 3420, 3365, 3321, 2959, 2210, 1622, 1544, 1503, 1465, 1415, 1331, 1252, 1126, 989, 841, 776, 744, $701 \mathrm{~cm}^{-1} .{ }^{1} \mathrm{H}$ NMR $(300$ MHz, DMSO-d $\left.{ }_{6}\right): \delta=7.77$ (bs, 2H, NH 2$), 7.51-7.59(\mathrm{~m}, 5 \mathrm{H}, \mathrm{Ar}-\mathrm{H}), 6.93(\mathrm{~s}, 2 \mathrm{H}, \mathrm{Ar}-\mathrm{H}), 3.82(\mathrm{~s}$, $\left.6 \mathrm{H}, \mathrm{OCH}_{3}\right), 3.76\left(\mathrm{~s}, 3 \mathrm{H}, \mathrm{OCH}_{3}\right)$. Anal. Calcd for $\mathrm{C}_{22} \mathrm{H}_{18} \mathrm{~N}_{4} \mathrm{O}_{3} \mathrm{~S}$ (418.47): C, 63.14; H, 4.34; N, 13.39. Found: C, 63.19; H, 4.28; N, 13.51.

\section{Acknowledgements}

The authors are thankful to the Department of Biotechnology, New Delhi for financial assistance.

\section{References}

1. (a) Caddick, S.; Fitzmaurice, R. Tetrahedron 2009, 65, 3325. (b) Kappe, C. O. Angew. Chem., Int. Ed. Engl. 2004, 43, 6250. (c) Dallinger, D.; Kappe, C. O. Chem. Rev. 2007, 107, 2563. (d) Chow, W. S.; Chan, T. H. Tetrahedron Lett. 2009, 50, 1286. 
2. (a) Dcmling, A.; Ugi, I. Angew. Chem. 2000, 112, 3300. (b) Domling, A.; Ugi, I. Angew. Chem., Int. Ed. 2000, 39, 3168. (c) Jimenez-Abnso, S.; Chavez, H.; Estevez-Braan, A.; Ravelo, A.; Feresin, G.; Tapia, A. Tetrahedron 2008, 64, 8938. (d) Ramon, D. J.; Yus, M. Angew. Chem., Int. Ed. 2005, 44, 1602. (e) Shi, D.; Ni, S.; Yang, F.; Ji, S. J Hetrocycl. chem. 2008, 45, 1275.

3. (a) Gerencser, J.; Dorman, G.; Darvas, F. QSAR Comb. Sci. 2006, 439. (b) Orru, R. V. A.; de Greef, M. Synthesis 2003, 1471. (c) Hulme, C.; Gore, V. Curr. Med. Chem. 2003, 10, 51. (d) Evans, B. E.; Rittle, K. E.; Bock, M. G.; DiPardo, R. M.; Freidinger, R. M.; Whitter, W. L.; Lundell, G. F.; Veber, D. F.; Anderson, P. S.; Chang, R. S. L.; Lotti, V. J.; Cerino, D. J.; Chen, T. B.; Kling, P. J.; Kunkel, K.A.; Springer, J. P.; Hirshfield, J. J. Med. Chem. 1988, 31, 2235.

4. (a) Boger, D. L.; Nakahara, S. J. Org. Chem. 1991, 56, 880. (b) Boger, D. L.; Kasper, A. M. J. Am. Chem. Soc. 1989, 111, 1517. (c) Reddy, T. R. K.; Mutter, R.; Heal, W.; Guo, K., Gillet, V. J.; Pratt, S.; Chen, B. J. Med.Chem. 2006, 49, 607. (d) Zhang, T. Y.; Stout, J. R.; Keay, J. G.; Scriven, E. F. V.; Toomey, J. E.; Goe, G. L. Tetrahedron 1995, 51, 13177.

5. (a) Fletcher, M. D.; Hurst, T. E.; Miles, T. J.; Moody, C. J. Tetrahedron 2006, 62, 5454. (b) Van Aken, K. J.; Lux, G. M.; Deroover, G. G.; Meerpoel, L.; Hoornaert, G. J. Tetrahedron 1994, 50, 5211.

6. (a) Movassaghi, M.; Hill, M. D. J. Am. Chem. Soc. 2006, 128, 4592. (b) Winter, A.; Risch, N. Synthesis 2003, 2667.

7. Thomas, A. D.; Asokan, C. V. Tetrahedron Lett. 2002, 43, 2273.

8. Tanaka, K.; Mori, H.; Yamamoto, M.; Katsumara, S. J. Org. Chem. 2001, 66, 3099.

9. Mashraqui, S. H.; Karnik, M. A. Tetrahedron Lett. 1998, 39, 4895.

10. Renslo, A. R.; Danheiser, R. L. J. Org. Chem. 1998, 63, 7840.

11. (a) Vijn, R. J.; Arts, H. J.; Green, R.; Castelijns, A. M. Synthesis 1994, 573. (b) Ahmed, S.; Baruah, R. C. Tetrahedron Lett. 1996, 37, 8231.

12. Komatsu, M.; Ohgishi, H.; Takamatsu, S.; Ohshiro, Y.; Agawa, T. Angew. Chem., Int. Ed. Engl. 1982, 21, 213.

13. Anabha, E. R.; Nirmala, K. N.; Thomas, A.; Asokan, C. V. Synthesis 2007, 428.

14. (a) Evdokimov, N. M.; Magedov, I. V.; Kireev, A. S.; Kornienko, A. Org. Lett. 2006, 8, 899.

(b) Evdokimov, N. M.; Kireev, A. S.; Yakovenko, A. A.; Antipin, M. Y.; Magedov, I. V.; Kornienko, A. J. Org. Chem. 2007, 72, 3443.

15. Ranu, B. C.; Jana, R.; Sowmiah, S. J. Org. Chem. 2007, 72, 3152.

16. Mamgain, R.; Singh, R.; Rawat, D. S. J. Heterocycl. Chem. 2009, 46, 69.

17. Sridhar, M.; Ramanaiah, B. C.; Narsaiah, C.; Mahesh, B.; Kumarswamy, M.; Mallu, K. K. R.; Ankathi, V. M.; Rao, P. S. Tetrahedron Lett. 2009, 50, 3897.

18. (a) Yamawaki, J.; Ando, T. Chem. Lett. 1979, 755. (b) Clark, J. H. Chem. Rev. 1980, 80, 429.

(c) Wang, X. S.; Zhang, M. M.; Li, Q.; Yao, C. S.; Tu, S. J. Synth. Commun. 2008, 38, 1896.

(d) Blass B. E. Tetrahedron 2002, 58, 9302. (e) Schmittling, E. A.; Sawyer, J. S. Tetrahedron Lett. 1991, 32, 7207. (f) Basu, B.; Das, P.; Das, S. Curr. Org. Chem. 2008, 12, 141. 